\title{
De la democracia en América Latina al comenzar el siglo XXI
}

\author{
MANUEL alCántARA SÁEZ
}

Catedrático de Ciencia Política de la Universidad de Salamanca

El presente trabajo reflexiona sobre el estado actual de la democracia en una región específica como es América Latina ${ }^{1}$ y los elementos que lastran su porvenir partiendo de diversas evidencias empíricas generadas por medio de diferentes indicadores entre los que los estudios de opinión pública de las masas y de las elites tienen un peso evidente. Se trata de una región que con mucha frecuencia queda alejada de los circuitos de gestación del pensamiento a pesar de constituir un escenario que aporta un material para el análisis y el estudio extremadamente rico. Ello se lleva a cabo en un momento en que parecen agostarse los ecos de la literatura académica de hace apenas una década que, tras agotar el estudio de las transiciones a la democracia, ponía el acento sobre la consolidación de las nuevas democracias, los factores que las articulaban y los retos que se cernian sobre ellas², para ser sustituidos por una nueva discusión sobre la calidad de la democracia en términos tanto de los mecanismos institucionales de la misma que producian su bonanza como del nivel de satisfacción de las expectativas generadas en sus "usuarios". Sin dejar de lado otras aproximaciones más o menos novedosas a la Ciencia Política desarrolladas en el puro ámbito latinoamericano como pudiera ser la articulada bajo el concepto de "democracia delegativa"”.

\section{Un diagnóstico de la política latinoamericana durante la decada DE 1990}

La política tiene un doble componente de búsqueda de mecanismos de consenso y de medios para afrontar el conflicto, reúne una serie de instrumentos de corte institucional para abordar y plantear soluciones para alcanzar el primero y superar el segundo. Desde esta perspectiva, los principales elementos de la agenda política de América Latina a lo largo de la década de 1990, en lo referido a la evolución de la democracia, permiten ser agrupados en cinco categorías de naturaleza diferente, pero que pretenden proyectar una imagen general de la región: se trata, en primer lugar, del mantenimiento de un mismo patrón de recambio 
democrático del liderazgo político; de la búsqueda, en segundo término, de mecanismos institucionales más idóneos para favorecer la acción del gobierno mediante los procesos de reforma política a través de cambios en el entramado constitucional; en tercer lugar, del mantenimiento de un tipo de Estado débil que, además, ha contemplado la elaboración de políticas tendentes a sustituir su histórico papel central en la economia por el del mercado; en cuarto lugar, del surgimiento de fenómenos populistas que, frente a modelos pretéritos, han optado por un marcado contenido antipolítico; $y$, por último, de un incremento de su posición marginal en la sociedad internacional como consecuencia del fin de la guerra fría y de la potenciación de otros nuevos escenarios. A continuación se desarrollan estos puntos.

Los procesos electorales han satisfecho correctamente sus funciones de recambio y/o de ratificación de la clase política como raramente y de forma tan generalizada con anterioridad habian hecho. Las elecciones se han llevado a cabo bajo prácticas de competencia libre, honesta e igualitaria, estando ausentes los otrora habituales mecanismos de fraude, fuera por la vía de la proscripción o de la manipulación del voto. Paralelamente, la alternancia de la clase política gracias a procedimientos constitucionales ha sido la norma, pudiéndose afirmar que los comicios se han convertido en el único medio legítimo sancionador del recambio politicos.

Durante la década de 1990 los países latinoamericanos han buscado de manera generalizada, mediante el rediseño de sus esquemas constitucionales, diferentes objetivos. De forma sucinta cabe referirse a seis ejes sobre los que han basculado las diferentes reformas políticas llevadas a cabo, reformas que, a veces, se han simultaneado en el mismo país. El primero, y más relevante, ha venido dirigido para asegurar el predominio del Poder Ejecutivo en el sistema político mediante la introducción de la reelección presidencial en Argentina, Brasil, Perú y Venezuela ${ }^{5}$, el incremento del periodo de mandato presidencial en Bolivia y Venezuela, el aumento de las facilidades para articular la legislación delegada, la parálisis hacia mayores formas de control desde el Poder Legislativo ${ }^{6}$, básicamente mediante el juicio político a presidentes ${ }^{7} \mathrm{o}$ a otros altos funcionarios. El segundo, en consonancia con el anterior, proyecta un Poder Legislativo más débil que ha perdido centralidad en el juego político como consecuencia de la tendencia registrada a articularlo en una sola cámara, como ocurrió tras las reformas constitucionales de Perú de 1993 y de Venezuela de $1999^{\circ}$. El tercero ha buscado una mayor legitimidad y operatividad de los procesos electorales. Para ello se ha incidido en cuatro niveles: la mejora de la administración electoral para asegurar la confiabilidad de las elecciones ${ }^{9}$; la extensión a un número mayoritario de casos del ballotage como forma electoral presidencial que dote a los candidatos electos de un mayor nivel de legitimidad ${ }^{10}$; la incorporación en la Constitución o 
en la legislación atinente a los partidos políticos de fórmulas de democracia interna tanto para su funcionamiento como para la elección de sus candidatos ${ }^{11}$ y el tímido avance en los mecanismos de control del gasto político junto con el paulatino incremento del peso de la financiación pública de la política. El cuarto eje ha redefinido el papel del Estado en la economía y en sus relaciones con el derecho de propiedad para permitir la introducción del modelo neoliberal ${ }^{12}$. El quinto ha continuado la tendencia de la década anterior proclive a la descentralización política y administrativa en busca de una mayor eficiencia y de una aproximación a los ciudadanos ${ }^{13}$; de esta manera se pusieron en marcha procesos de elección popular de autoridades locales ${ }^{14}$ y se potenciaron las figuras de las entidades territoriales ya existentes. Finalmente el sexto eje ha supuesto la incorporación de nuevas figuras institucionales en los ordenamientos juridicos latinoamericanos que van desde la introducción en los textos constitucionales de organismos tendentes a la organización, administración y gobierno del Poder Judicial, o de la defensoría del pueblo, al reconocimiento del carácter pluricultural y multirracial de las sociedades ${ }^{15}$.

El Estado latinoamericano ha profundizado su histórica debilidad a lo largo de la década de 1990. A sus tradicionales carencias de poder tener el monopolio de la violencia legitima, de control del territorio ${ }^{16}$ y de construcción de una ciudadanía portadora de valores civicos y republicanos y sujeto de derechos universales con capacidad plena para ejercerlos ${ }^{17}$, se debe incorporar el legado del consenso de Washington ${ }^{18}$. Dicho consenso impuso la austeridad fiscal, en lo atinente, fundamentalmente, a la constricción del gasto público, la privatización de activos públicos, la liberalización de los mercados y la desregulación, aspectos todos ellos que limaron la ya de por si reducida capacidad operativa del Estado latinoamericano. En términos generales, el Estado dejó de tener capacidad operativa para ser el implementador de políticas públicas que pudiera diseñar el gobierno para alcanzar ciertos objetivos programáticos acordes con las reivindicaciones y necesidades de las sociedades latinoamericanas.

América Latina ha registrado, igualmente, la continuidad de expresiones populistas que se creian periclitadas y que se han producido en un numero relativamente bajo de países. La novedad, sin embargo, ha sido la aparición de fórmulas imbuidas por su carácter desmovilizador y reivindicador de comportamientos antipoliticos. Frente al populismo exhibido por Carlos S. Menem, fuertemente arropado por la maquinaria política del histórico Partido Justicialista o el de Abdalá Bucaram asido al Partido Roldosista Ecuatoriano, se suscitó el de Alberto Fujimori quien diseñó una actuación destinada a alejar a la política de su acción de gobierno para lo cual desdeñó la movilidad social y/o política que pudo haber articulado mediante algún movimiento o partido, pero que renunció expresamente a ello y despolitizó todas aquellas otras instancias en que pudieran quedar res- 
coldos de la hoguera política precedente: de esta manera desvirtuó y redujo a la más minima expresión el papel del Congreso, pero también de las Municipalidades que quedaron convertidas en oficinas de gestión técnica de proyectos varios. En un espacio intermedio, Hugo Chávez se alza como el populista fustigador de la clase politica anterior, pero frente a la partidocracia de entonces no renuncia a crear su propio aparato movilizador y a fortalecer el instrumento institucional en el que se ha apoyado en los últimos años, sin dejar el carácter personalista, de discurso emotivo y bañado en miticas referencias cargadas de fuerte simbolismo que imbuye a su acción política.

Finalmente, América Latina ha contemplado a lo largo de la década de 1990 cómo su posición en el escenario internacional se ha ido marginando. Un problema que siempre ha tenido la región ha sido su carácter heterogéneo, sus intereses variopintos y su liderazgo disperso. Todos estos factores han imposibilitado la existencia de una única voz producto de posturas mínimamente comunes, lo cual ha generado cierta inconsistencia e incluso contradicción en su posición que ha proyectado una imagen de debilidad de su presencia en el foro internacional'. Por otra parte, la caida del muro de Berlin eliminó el referente del socialismo real para amplios sectores de la izquierda, redujo a la mínima expresión los apoyos procedentes de aquel espacio, hizo desaparecer en el imaginario de Washington la idea de que su "patio trasero" pudiera caer en manos enemigas, redujo su peso especifico en los organismos internacionales en los que la tradicional alineación con las posturas de Estados Unidos le hacia gozar de una timida posición "de chantaje", y desplazó la centralidad de la política hacia el este de Europa, bien por los procesos de transición politica y económica que se suscitaron, bien por el estallido del conflicto de los Balcanes. Paralelamente, América Latina siguió perdiendo peso en la economía mundial como consecuencia del hundimiento al que habia llegado su modelo económico establecido medio siglo atrás, del mayor dinamismo de las economías del sudeste asiático y de la atención que requería la más dramática situación, en términos de demanda de ayuda, del Africa subsahariana. Si bien los lazos de institucionalización formal con el norte del continente dieron paso a la primera Cumbre de las América en 1994 y cinco años más tarde a la primera Cumbre Unión Europea, América Latina y Caribe, los resultados no fueron muy boyantes ${ }^{20}$. América Latina apenas si ocupaba dos renglones en la agenda internacional referidos al narcotráfico y a la emigración. únicos aspectos con capacidad de impacto y de cierta respuesta en Estados Unidos ${ }^{21}$ y la Unión Europea.

\section{SOBRE LA CALIDAD DE LA DEMOCRACIA EN AMÉRICA LATINA AL COMIENZO DEL SIGLO XXI}

El nuevo elemento que ha supuesto la expansión de los derechos políticos contrasta con el mantenimiento de viejos patrones en lo relativo al déficit de dere- 
chos civiles básicos y de derechos sociales en los sectores populares, aspectos que no son sino el reflejo de las profundas desigualdades que imperan en la región" y de los efectos de la aguda crisis económica que la ha asolado a lo largo de buena parte de la década de 1990. Diferentes instrumentos para medir la situación politica en un momento dado que pueden recoger su dinamismo a lo largo de un determinado periodo ponen de reflejo la enorme variación que se da entre los paises latinoamericanos y que a veces tiende a ser ocultada por aproximaciones demasiado generales realizadas desde una supuesta perspectiva regional. Las clasificaciones que estos instrumentos permiten establecer (ver Cuadros I y II) son funcionales para, una vez determinado el orden, poder diseñar una tipologia de paises "democráticamente más avanzados". "intermedios" y paises "democráticamente menos avanzados". Con ello pueden establecerse desde ta enunciación de las principales caracteristicas de sus sistemas politicos los elementos que mejor pueden incidir en la calidad de su politica.

La combinación de las clasificaciones de Fitzgibbor ${ }^{3}$ y de Freedom House permiten hacer la citada triple clasificacion de manera que aparecen como los paises latinoamericanos democráticamente más avanzados: Argentina. Brasil. Costa Rica, Chile, México, República Dominicana y Uruguay; los Intermedios: Bolivia. Colombia, Ecuador. El Salvador, Honduras, Nicaragua. Panamá. Perú y Venezuela; y los democràticamente menos avanzados: Cuba, Guatemala, Haiti y Paraguay.

Cuadro 1

\section{Clasificación de las democracias latinoamericanas en el año 2000 de acuerdo con la encuesta Fitzgibbon}

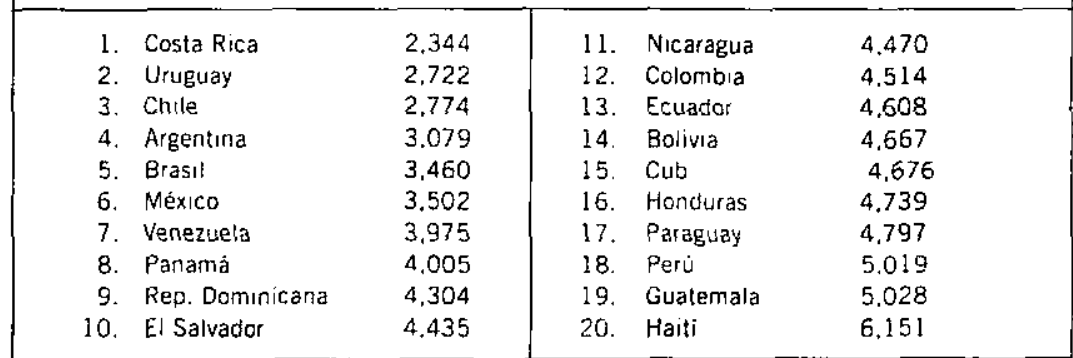

Fuente: Lasa Forum. Vol XXXill. I. primavera de 2002. Pàg. 12

Ahora bien, estas clasificaciones pueden ser complementadas mediante las percepciones que de la politica tienen los actores de América Latina y que tienen que ver con el énfasis dado al papel que las aspiraciones democráticas de la ciudadania desempeñan en relación con la calidad de la política. Dichas aspiraciones, en un primer momento, se confunden con la legitimidad democrática que poseen un alto componente subjetivo según el cual los individuos creen que un 
Cuadro 2

\section{Evolución de la clasificación de los países latinoamericanos de acuerdo con los índices de Freedom House}

\begin{tabular}{|l|l|l|l|l|l|l|l|l|l|l|l|l|l|l|}
\hline \multicolumn{1}{|c|}{ Pais } & $1976-77$ & $1990-91$ & $2001-02$ & País & \multicolumn{2}{|l|}{$1976-77$} & \multicolumn{2}{|c|}{$1990-91$} & \multicolumn{2}{|c|}{$2001-02$} \\
\hline & DP & LC & DP & LC & DP & LC & & DP & LC & DP & LC & DP & LC \\
\hline Argentina & 6 & 5 & 1 & 3 & 2 & 3 & Haiti & 6 & 6 & 4 & 4 & 6 & 6 \\
Bolivia & 6 & 4 & 2 & 3 & 1 & 3 & Honduras & 6 & 3 & 2 & 3 & 3 & 3 \\
Brasil & 4 & 5 & 2 & 3 & 3 & 3 & México & 4 & 4 & 4 & 4 & 2 & 3 \\
Colombia & 2 & 3 & 3 & 4 & 4 & 4 & Nicaragua & 5 & 5 & 3 & 3 & 3 & 3 \\
Chile & 7 & 5 & 2 & 2 & 2 & 2 & Panamá & 7 & 6 & 4 & 2 & 1 & 2 \\
Costa Rica & 1 & 1 & 1 & 1 & 1 & 2 & Paraguay & 5 & 6 & 4 & 3 & 4 & 3 \\
Cuba & 7 & 6 & 7 & 7 & 7 & 7 & Perú & 6 & 4 & 3 & 4 & 1 & 3 \\
Ecuador & 6 & 5 & 2 & 2 & 3 & 3 & R.Dominicana & 4 & 3 & 3 & 3 & 2 & 2 \\
El Salvador & 3 & 3 & 3 & 4 & 2 & 3 & Uruguay & 6 & 6 & 1 & 2 & 1 & 1 \\
Guatemala & 4 & 3 & 3 & 4 & 3 & 4 & Venezuela & 1 & 2 & 1 & 3 & 3 & 5 \\
\hline
\end{tabular}

Fuente: Freedom in the World: The Annual Survey of Political Rights and Civil Liberties. Londres. Freedom House. 2002 DP: Derechos Politicos: LC: Libertades Civiles

El rango supone la categoria de "paises libres" a los que situan su promedio entre I y 3; "paises parcialmente libres" a los que lo situan entre 3 y 5,5; y "no libres" a los que lo situan entre 5,5 y 7.

determinado sistema, unas reglas procedimentales o un conjunto de politicas son objeto de aceptación y de obediencia y, más aún, son considerados como capaces de resolver sus problemas más inmediatos y relevantes. Si bien en un primer momento la democracia es evaluada per se, como el mecanismo institucional más idóneo, respetable y valioso en sí mismo, con el paso del tiempo la acción politica se confunde con el ámbito más genérico que define a las reglas y que no es sino la democracia. El desgaste del gobierno de turno, de sus politicas, y todos sus desarreglos terminan por apoderarse asimismo del àmbito donde aquéllas se llevan a cabo.

En América Latina el último lustro ha supuesto un fuerte deterioro de las economias nacionales que ha llevado a sus ciudadanos a cotas preocupantes de precariedad en sus condiciones de vida cotidiana y que, junto con el crecimiento del conocimiento de los escándalos de corrupción politica, ha contribuido a un fuerte descenso de la credibilidad de la politica sin que por ello necesariamente hayan surgido ofertas no democráticas que gozaran de apoyo manifiesto por parte de la ciudadania ni hayan debilitado el progreso indudable que con respecto al pasado inmediato goza el apoyo a la democracia. En términos eastonianos ${ }^{24}$ se ha seguido manteniendo un apoyo difuso que garantiza legitimidad a la democracia. Ello no ha impedido que, de forma puntual, la percepción de los latinoamericanos sobre la preferencia de la democracia frente a otra forma de gobierno haya sufrido un retroceso que, con matices, puede considerarse generalizado en 2002 con respecto a los años precedentes (véase Cuadro III). Los datos de la evolución 
de las preferencias de los ciudadanos muestran un inequivoco descenso en la mayoria de los paises en la valoración de la democracia donde solamente México y Venezuela poseen los valores más altos de preferencia de la democracia como forma de gobierno en 2002 de entre las series temporales de ambos paises. Si en 1996 siete de los diecisiete paises considerados tenian valores favorables a la preferencia de la democracia iguales o superiores al sesenta por ciento y lodos menos dos (Honduras y Brasil) tenian valores superiores ai cincuenta por ciento. en 2002 eran seis los paises que mantenian valores superiores al sesenta por ciento y otros seis valores inferiores al cincuenta por ciento. En el polo opuesto, las preferencias a favor de un gobierno autoritario han descendido sensiblemente, ya que si en 1996 cinco paises tenian valores de apoyo a este tipo de gobierno en porcentajes de su población superiores o iguales al veinte por ciento, en 2002 dicho número habia descendido a tres.

Por otra parte. las series de respuestas de los ciudadanos en el periodo considerado muestran un declive más pronunciado de las preferencıas democráticas en torno a los quince puntos porcentuales en seis paises: Brasil. Colombia, El Salvador, Nicaragua, Panamá y Paraguay. Sin embargo, las preferencias autoritarias no han crecido en proporción paralela. De hecho, en la mayoria de los casos se han mantenido e incluso han disminuido. Unicamente se ha registrado un incremento importante en Panamá y, sobre todo. Paraguay. Ello conduce a mantener la existencia de una bolsa relevante de población superior a una cuarta parte de la población entrevistada en un buen número de paises (diez) que sostiene una posición tercera reflejada en la idea de que "a la gente como yo nos da lo mismo el tipo de régimen político existente". Esta población apática es el prıncipal efecto de la crisis de la década de 1990 y puede considerarse como un "ejército de reserva" para acompañar cualquier tipo de aventura politica que se pudiera poner en marcha.

Los paises latinoamericanos que destacan por su mayor nivel de preferencia de la democracia a cualquier otra forma de gobierno por parte de la población entrevistada y que, a su vez, mantienen valores estables a lo largo del periodo 1996 2002 con una media superior al sesenta por ciento son Argentina, Costa Rica, Honduras, Nicaragua, Panamá, Perú. Uruguay y Venezuela, de los que destacan sobremanera Costa Rica y Uruguay con valores constantes en torno al achenta por ciento, lo que viene a suponer una categoria especial. Por el contrario, los paises que se situan en el rango de menor nivel de preferencia, con valores medios inferiores al cincuenta por ciento son Ecuador, Guatemala y Paraguay. Brasil, Colombia y El Salvador son paises sin preferencia por el tipo de gobierno, actitud más constante en los dos primeros, donde el cinismo politico tiene mayor presencia, que en el tercero puesto que esta tendencia no se hace patente sino en 2001. 


\section{Cuadro 3}

\section{Preferencias sobre formas de gobierno \\ ¿Con cuál de las siguientes afirmaciones está usted más de acuerdo? (\%)}

\begin{tabular}{|l|c|c|c|c|c|c|c|c|c|c|c|c|}
\hline & \multicolumn{5}{|c|}{$\begin{array}{c}\text { La democracia es preferible a cualquier } \\
\text { otra forma de gobierno }\end{array}$} & \multicolumn{5}{c|}{$\begin{array}{r}\text { En algunas circunstancias es preferible } \\
\text { un gobierno autoritario }\end{array}$} \\
\hline & 1996 & 1997 & 1998 & 2000 & 2001 & 2002 & 1996 & 1997 & 1998 & 2000 & 2001 & 2002 \\
\hline Argentina & 71 & 75 & 73 & 71 & 58 & 65 & 15 & 15 & 16 & 16 & 21 & 17 \\
\hline Bolivia & 64 & 66 & 55 & 62 & 54 & 56 & 17 & 16 & 22 & 13 & 17 & 20 \\
\hline Brasil & 50 & 50 & 48 & 39 & 30 & 37 & 24 & 19 & 18 & 24 & 18 & 15 \\
\hline Chile & 54 & 61 & 53 & 57 & 45 & 50 & 19 & 16 & 16 & 19 & 19 & 14 \\
\hline Colombia & 60 & 69 & 55 & 50 & 36 & 39 & 20 & 13 & 17 & 23 & 16 & 11 \\
\hline Costa Rica & 80 & 83 & 69 & 83 & 71 & 77 & 7 & 9 & 21 & 6 & 8 & 8 \\
\hline Ecuador & 52 & 41 & 57 & 54 & 40 & 49 & 18 & 23 & 19 & 12 & 23 & 18 \\
\hline El Salvador & 56 & 66 & 79 & 63 & 25 & 40 & 12 & 13 & 10 & 10 & 10 & 16 \\
\hline Guatemala & 51 & 48 & 54 & 45 & 33 & 45 & 21 & 26 & 29 & 21 & 21 & 12 \\
\hline Honduras & 42 & 63 & 57 & 64 & 57 & 57 & 14 & 17 & 9 & 15 & 8 & 14 \\
\hline México & 53 & 52 & 51 & 45 & 46 & 63 & 23 & 31 & 28 & 34 & 35 & 20 \\
\hline Nicaragua & 59 & 68 & 72 & 64 & 43 & 63 & 14 & 19 & 9 & 6 & 22 & 12 \\
\hline Panamán & 75 & 71 & 71 & 62 & 34 & 55 & 10 & 10 & 8 & 18 & 23 & 16 \\
\hline Paraguay & 59 & 44 & 51 & 48 & 35 & 45 & 26 & 42 & 36 & 39 & 43 & 38 \\
\hline Perú & 63 & 60 & 63 & 64 & 62 & 57 & 13 & 16 & 12 & 13 & 12 & 16 \\
\hline Uruguay & 80 & 86 & 80 & 84 & 79 & 78 & 9 & 7 & 9 & 9 & 10 & 10 \\
\hline Venezuela & 62 & 64 & 60 & 61 & 57 & 75 & 19 & 17 & 25 & 24 & 20 & 12 \\
\hline
\end{tabular}

Fuente: Latinobarómetro. The Economist, 28 de julio de 2001 y 15 de agosto de 2002.

Como complemento a la evolución de las preferencias populares sobre el tipo de gobierno existente en su pais puede considerarse la opinión de la clase politica acerca del grado de estabilidad que la democracia tiene en su pais (véase Cuadro $(V)^{25}$. Si bien se trata ahora de evaluaciones de un sector de la clase politica con obvios supuestos mentales diferentes a los de la generalidad de la población, su percepción de la vida política en términos agregados, por la posición que ocupan, es valiosa. De esta manera, y dentro de los dieciséis casos de los que se tienen datos, pueden establecerse tres grupos de paises que son un reflejo de las profundas diferencias existentes entre los mismos: Ios de alta estabilidad (Argentina, Chile, Costa Rica y Uruguay) Ios de estabilidad media (Bolivia, Colombia. Honduras y República Dominicana) y los de baja estabilidad (Ecuador, El Salvador, Guatemala, México, Nicaragua, Paraguay, Perủ y Venezuela).

La integración en una única clasificación ${ }^{26}$, de acuerdo con los dos criterios recién enunciados, proyecta el escenario que se presenta en el Cuadro V. Los dieciséis casos nacionales recogidos cubren nueve de las doce casillas definidas lo cual es un indicador que refleja la validez de las taxonomias establecidas. A pesar de la severa crisis que asola Argentina desde finales de 2001, este pais, junto con Costa Rica y Uruguay se sitúa en una posición de máxima bonanza demo- 
Cuadro 4

\section{El grado de estabilidad de la democracia}

\begin{tabular}{|c|c|c|c|c|c|c|c|c|}
\hline País & Congreso & Muy estable & $\begin{array}{c}\text { Bastante } \\
\text { estable }\end{array}$ & $\begin{array}{c}\text { Poco } \\
\text { estable }\end{array}$ & $\begin{array}{c}\text { Nada } \\
\text { estable }\end{array}$ & N.S & N.C & (N) \\
\hline Argentina & $(95-97)$ & 49,7 & 47,0 & 3,2 & 0 & 0 & .0 & (68) \\
\hline \multirow[b]{2}{*}{ Bolivia } & $(97-01)$ & 59,4 & 34,4 & 3,9 & , 0 & 0 & 2,3 & (128) \\
\hline & $(97-02)$ & 27,6 & 57,1 & 14,3 & 1,0 & .0 &, 0 & (98) \\
\hline \multirow[t]{2}{*}{ Chile } & $(93-97)$ & 32,7 & 64,3 & 3,0 & .0 & 0 & , 0 & (93) \\
\hline & $(97-01)$ & 33,7 & 62,9 & 3,4 & , 0 & 0 &, 0 & (89) \\
\hline \multirow{3}{*}{$\begin{array}{l}\text { Colombia } \\
\text { Costa Rica }\end{array}$} & $(98-02)$ & 20,9 & 61,0 & 16,0 & 2,2 &, 0 &, 0 & (88) \\
\hline & $(94-98)$ & 61,5 & 38,5 & ,0 & .0 & .0 & 0 & (52) \\
\hline & (98-02) & 46,9 & 44,9 & 8,2 & 0 &, 0 & .0 & (49) \\
\hline Ecuador & $\{96-98\}$ & 11,3 & 76,7 & 6,6 & 2,9 & .0 & 2,5 & $\langle 72\rangle$ \\
\hline \multirow[b]{2}{*}{ El Salvador } & $(98-02)$ & 5,4 & 49,5 & 35,1 & 7,2 & , 0 & 2,7 & (111) \\
\hline & $(94-97)$ & 4,6 & 44,4 & 36,9 & 6,3 & 1,7 & 6,1 & (46) \\
\hline \multirow[b]{3}{*}{ Guatemala } & $(97-00)$ & 7,4 & 51,6 & 35,8 & , 0 & .0 & 5,2 & (58) \\
\hline & $(00-03)$ & 19,5 & 36,1 & 41,6 & 0 &, 0 & 2,8 & (64) \\
\hline & (95-99) & 17,5 & 30,2 & 46,0 & 3,2 & 0 & 3,2 & (63) \\
\hline Honduras & $(94-97)$ & 19,8 & 62,7 & 14,4 &, 0 & 3,0 & 0 & (67) \\
\hline \multirow[b]{2}{*}{ Nicaragua } & $(97-01)$ & 29,6 & 54,9 & 15,5 & 0 & 0 & 0 & (71) \\
\hline & $(96-01)$ & 4,3 & 27,1 & 57,1 & 10,0 & 1,4 & .0 & (70) \\
\hline México & $(94-97)$ & 10,3 & 26,0 & 32,3 & 26,3 & ,6 & 4,5 & (123) \\
\hline \multirow[b]{3}{*}{ Paraguay } & $(97-00)$ & 9,5 & 38,1 & 44,4 & 6,3 & 1,6 & , 0 & (126) \\
\hline & $(00-03)$ & 15,7 & 48,8 & 33,1 & 2,5 & 0 & 0 & (121) \\
\hline & (93-98) & 4,3 & 37,0 & 56,7 & 2,0 & .0 & 0 & (47) \\
\hline \multirow[b]{2}{*}{ Perú } & $(98-03)$ & 1,5 & 40,0 & 52,3 & 6,2 & , 0 & 0 & (65) \\
\hline & $(01-06)$ & 5,1 & 26,5 & 62,5 & 3,8 & .0 & 2,2 & (83) \\
\hline R. Dominicana & (94-98) & 30,8 & 22,3 & 35,0 & 8,7 & 0 & 3,3 & $\langle 62\rangle$ \\
\hline \multirow[b]{2}{*}{ Uruguay } & $(98-02)$ & 46,6 & 44,7 & 8,7 & , 0 & 0 & , 0 & (103) \\
\hline & $(00-05)$ & 66,5 & 29,5 & 4,0 & .0 & 0 & 0 & (68) \\
\hline Venezuela & $(00-05)$ & 17,8 & 45,3 & 26,3 & 3,9 & 0 & 6,6 & $(100)$ \\
\hline
\end{tabular}

Pregunta En su opinión, ¿la democracia en su pais es hoy: muy estable, bastante estable, poco estable o nada estable? Fuente: Proyecto Elites Parlamentarias Latinoamericanas. Salamanca 1994-2005.

crática. Esta es una posición que mantiene una clara disonancia con el repudio manifiesto hacia la clase politica argentina. Mientras que la democracia parece alcanzar cotas de gran vigor, la politica, como actividad cotidiana registra el rechazo masivo de los ciudadanos ${ }^{27}$. De esta forma, la decepción con respecto al liderazgo político se ha convertido en el principal factor de debilitamiento de la percepción de estabilidad de la democracia en condiciones en que se sigue manteniendo una clara preferencia por la misma. El cuestionamiento del liderazgo del peruano Alejandro Toledo ${ }^{28}$, la polarización en torno al liderazgo del venezolano Hugo Chávez y la disputa entre el nuevo presidente de Nicaragua Bolaños y el saliente Arnoldo Alemán pueden estar en la base de la explicación de la ubicación de estos tres países en un espacio con obvias categorías disonantes de alta preferencia por el gobierno democrático y bajo grado de percepción de estabilidad de la democracia. 
Cuadro 5

\section{Los países latinoamericanos de acuerdo con sus preferencias ante un gobierno democrático y a la estabilidad de la democracia}

\begin{tabular}{|l|c|c|c|}
\hline & $\begin{array}{c}\text { Paises de alto grado } \\
\text { de percepción } \\
\text { de estabilidad de la democracia }\end{array}$ & $\begin{array}{c}\text { Paises de grado medio } \\
\text { de percepción } \\
\text { de estabilidad de la democracia }\end{array}$ & $\begin{array}{c}\text { Países de bajo grado } \\
\text { de percepción } \\
\text { de estabilidad de la democracia }\end{array}$ \\
\hline $\begin{array}{l}\text { Países con clara preferencia } \\
\text { por el gobierno democrático* }\end{array}$ & $\begin{array}{c}\text { Argentina } \\
\text { Costa Rica } \\
\text { Uruguay }\end{array}$ & $\begin{array}{c}\text { Honduras } \\
\text { República Dominicana }\end{array}$ & $\begin{array}{c}\text { Perú } \\
\text { Venezuela }\end{array}$ \\
\hline $\begin{array}{l}\text { Países con leve preferencia } \\
\text { por el gobierno democrático }\end{array}$ & Chile & Bolivia & $\begin{array}{c}\text { Ecuador } \\
\text { México } \\
\text { Guatemala }\end{array}$ \\
\hline $\begin{array}{l}\text { Países con mayor preferencia } \\
\text { por el gobierno autoritario }\end{array}$ & & Paraguay \\
\hline $\begin{array}{l}\text { Paises sin preferencia por el } \\
\text { tipo de gobierno** }\end{array}$ & Colombia & El Salvador \\
\hline
\end{tabular}

- Aqui se encontraria Panamá pero no hay datos de Elites Parlamentarias sobre su grado de estabilidad.

* Aqui se encontraria Brasil pero no hay datos de Elites Parlamentarias sobre su grado de estabilidad.

Fuente: Elaboración propia

Otro factor a ser considerado por su notable impacto en la calidad de la politıca es la corrupción. Medida desde las percepciones que tienen diferentes actores en contacto con las realidades políticas nacionales latinoamericanas, la corrupción tiene un nivel de presencia muy diferente en la región y sus efectos son claramente identificables. Los tres paises latinoamericanos que se sitúan en la parte alta de la clasificación establecida con respecto al İndice de Percepción de Corrupción ${ }^{29}$ : Chile, Uruguay y Costa Rica están asimismo al frente de una supuesta clasificación en la calidad de su politica.

\section{CONCLUSIONES}

La democracia se encuentra asentada en América Latina como nunca antes en su historia en términos de continuidad y de presencia en mayor número de paises. La perdurabilidad de la misma a lo largo del último cuarto de siglo es consecuencia fundamentalmente de la extensión del propio credo democrático entre diferentes actores relevantes que han interiorizado sus valores e incluso adoptado una posición de promoción de los mismos. Empresarios, militares, nuevos movimientos sociales y la clase politica han asumido la democracia como "el único juego posible" ${ }^{30}$. Pero también el favorable escenario internacional ha sido un elemento de indudable impacto. Estas dos circunstancias han contrastado con el pobre rendimiento de la economia cuyo comportamiento no ha contribuido, como los teóricos de la teoría de la modernización habrian esperado, a su asentamiento ${ }^{3 !}$. 
El asentamiento de la democracia en América Latina no es óbice para que el desempeño de la política suponga constantes situaciones que, con frecuencia, generan malestar entre los ciudadanos como consecuencia de la no resolución de sus problemas más acuciantes o incluso de la extensión de la convicción de que ellos ni siquiera son abordados por las autoridades competentes. En cierta medida ello es consecuencia de diseños institucionales propios entre los que destacan la forma de gobierno presidencialista, las peculiares relaciones conformadas en el triángulo compuesto por el Poder Ejecutivo, el Poder Legislativo y los partidos políticos, pero también lo es a causa de determinados elementos constitutivos de una cultura política definida por cotas alarmantes de desconfianza en lo público y en las distintas maneras de ejercer la autoridad en dicho ámbito, y, finalmente, se encuentra el menor espacio dejado por el entramado internacional que apenas si permite una mínima autonomía para la toma de decisiones propias. Así mismo se halla la necesaria redefinición del papel del Estado ninguneado por el avance del denominado neoliberalismo articulado en clave de "pensamiento único". El Estado aparece ante diferentes sectores sociales o ámbitos territoriales en un escenario ambiguo definido tanto en lo relativo a su histórica y manifiesta ausencia y en su incapacidad para aplicar el monopolio legítimo de la fuerza como en sus relaciones con la sociedad en el reino de la economía. Espacio al que tampoco ha sido ajena la agudización reciente de los procesos de globalización más activos, si cabe, en dicho terreno de la economia.

Todo ello no es sino un patrón de normalización de la política en América Latina, como nunca antes había ocurrido en su historia. El conflicto, el disenso y la mejor o peor calidad de la implementación de decisiones políticas con sus correspondientes efectos, esperados o no, son aspectos de la vida cotidiana que, a su vez, se ve inmersa en dificultades en el avance de los ciudadanos como agentes en un marco regido por las reglas de la poliarquía. La institucionalización de la política, de acuerdo con pautas poliárquicas, es una evidencia de la realidad latinoamericana que afronta un futuro en condiciones muy diferentes a las de hace un cuarto de siglo donde, como ha quedado de manifiesto en las páginas anteriores, se da un escenario de claroscuros.

Se trata no sólo de que el juego político funcione mediante reglas democráticas sino también que éstas penetren en el interior de las mismas. La demanda ahora no se basa en la libertad de la competencia partidista real, ni en las elecciones a órganos colegiados o no de gobierno y de control, se proyecta en la imponderable articulación democrática en el interior de los mismos, en la democracia interna en los partidos, en los parlamentos y en las reglas de relación entre diferentes poderes. Además, se trata de incorporar a sectores marginales que, en unas ocasiones, acarrean siglos de marginación, pero que en otras son consecuencia del desarrollo desigual. Estos sectores ignoran con frecuencia las reglas de la poliar- 
quía o incluso mantienen comportamientos claramente de anomia y están completamente huérfanos de capital político ${ }^{32}$.

El funcionamiento de las instituciones se alza como un reto fundamental en el devenir de la politica. Unas instituciones con mecanismos de actuación concretos que, muchas veces, producen efectos diferentes a los inicialmente esperados o que, en otras ocasiones, han sido incorporadas sin tener en cuenta la realidad del lugar de su ubicación. La atención más que nunca a estos extremos es un imperativo obligatorio en el desempeño de la política. Paralelamente, el comportamiento de los actores, enmarcados en roles y posibilidades de actuación en la politica novedosas, supone un frente de inquietudes que no debe ignorarse aunque en muchos casos se trate de actores que continúan incorporando el legado del pasado. Por último, la internacionalización de problemas, hasta hace poco considerados como locales, que comportan soluciones que rompen principios clásicos basados en la soberanía nacional ha introducido un sesgo distinto en la politica latinoamericana muy diferente al escenario vislumbrado en las décadas de 1960 y de 1970 por los seguidores de la Teoría de la Dependencia.

La democracia en el último cuarto de siglo en América Latina superó una primera etapa en la que la oleada de transiciones fue su elemento definitorio ${ }^{33}$. Durante la misma se evitó el primer desafío que representaban las involuciones autoritarias, la economía gozó de cierta estabilidad lográndose una relativa distribución de la riqueza y se asentaron las reglas de juego democráticas como consecuencia, sobretodo, de acuerdos entre las elites. Posteriormente pasó por una segunda etapa en la que el elemento protagonista fue la transición económica como consecuencia de la quiebra de la matriz estado-céntrica. Mientras que el desafío político de ese momento consistió en la derrota de los intereses articulados en torno al Estado, el desafío económico giró alrededor del ajuste y de la reforma estructural orientada hacia el mercado. En esta etapa los partidos se asentaron y se rutinizaron diferentes mecanismos institucionales de la poliarquía que, no obstante, mantuvieron cierta debilidad. La reestructuración económica trajo consigo serios costos sociales y se terminaron imponiendo liderazgos autoritarios de nuevo cuño. La tercera etapa en la que se entra al final de la década de 1990 tiene como principales desafíos el fortalecimiento institucional y el crecimiento económico sostenido con distribución. Las amenazas proceden de desarreglos en el interior de las instituciones como consecuencia de deficiencias en su funcionamiento y debilidades de la organización y de retos exógenos a las instituciones en la esfera de la sociedad, como serían los estragos de la pobreza y los efectos psicológicos de la inseguridad ciudadana, y en el ámbito internacional como consecuencia de la política autista de Estados Unidos. Sendas amenazas podrían tener efectos en el medio y largo plazo sobre una cultura política en la que perviven rasgos de paternalismo y de clientelismo alimentando una reacción de la ciuda- 
danía que abogase por soluciones en clave de antipolítica ${ }^{34}$ como las que de manera salpicada se han ido dando principaimente en los países andinos a 10 largo de los dos últimos lustros.

\section{BIBLIOGRAFÍA CITADA}

Alcántara, Manuel (1991). "Sobre el concepto de paises en vias de consolidación democrática en América latina". Revista de Estudios Politicos. 74. Octubre-Diciembre. Madrid: Centro de Estudios Constitucionales. Págs. 113-130.

Alcántara, Manuel (1992). "¿Democracias inciertas o democracias consolidadas en América Latina?". Revista Mexicana de Sociologia.54.1. Págs. 205-223.

Alcántara, Manuel (2001). "Experimentos de democracia interna: Las primarias de partidos en América Latina". Papeles de Trabajo. México: FLACSO

Alcántara, Manuel (2002). ¿Institutiones o máquinas ideológicas? Origen, programay organización de los partidos políticos latinoamericanos. Madrid. Istmo.

Easton, David (1975). "A Re-assessment of the Concept of Political Support". British Journal of Political Science. 5. Págs. 435457.

Jordana, Jacint (2001). Relaciones intergubernamentales $y$ descentralización en América Latina: una perspectiva institucional. Washington. Banco Interamericano de Desarrollo. Serie de Documentos de Trabajo 1-22UE.

Kenney, Charles (1997). "Anti-Políticos, Intrusos y Política Democrática". E politiké. Morón. 3. Págs. 28-42.

Linz, Juan J. (1990). "Transiciones a la democracia". Revista Española de Investigaciones Sociológicas. Madrid. 51.

Págs. 9-33.

Mainwaring, Scott (1999). "La durabilidad de la democracia en América Latina". Política y Gobierno. VI.2. Págs. 315-363.

Mateos, Araceli (2002). Cultura política y participación: las subculturas de los electores de 2000. Tesis Doctoral. Salamanca. Universidad de Salamanca.

Mayorga, René (1995). Antipolitica y neopopulismo. La Paz. CEBEM.

Méndez, Juan E. ; Guillermo O'Donnell y Paulo Sérgio Pinheiro (eds.) (1999). The (Un)Rule of Law \& the Unprivileged in Latin America. Notre Dame. University of Notre Dame Press.

Molina, Josè Enrique (2000). Los sistemas electorales de América Latina. Cuadernos de Capel 45. San José de Costa Rica.

Morgenstern, Scott y Benito Nacif (eds.) (2002). Legislative Politics in Latin America. Cambridge. Cambridge University Press.

O'Donnell, Guillermo (1994). "Delegative Democracy". En Journal of Democracy, 5.1. Págs. 55-69

O'Donnell, Guillermo (2002). "Human Development, human rights and democracy". Documento de Trabajo 1. Taller "Calidad de la democracia y desarrollo humano en América Latina". Heredia. Costa Rica. http://www.estadonacion.or.cr

Pérez Liñán, Anibal (2000). "¿Juicio político o golpe legislativo? Sobre las crisis constitucionales en los años noventa". En América Latina hoy, 26. Págs.: 67-74.

Tanaka, Martin (1999). "La consolidación de la democracia en América Latina y la importancia de la competencia politica: lecciones desde la experiencia peruana". Occasional Paper. The Japan Center for Area Studies. Suita. $N^{\circ} 4$.

Williamson, John (1994). The Political Economy of Policy Reform. Washington. Institute of International Economics. 


\section{NOTAS}

1. En otro trabajo he analizado el papel de los partidos politicos. Véase Alcántara (2002).

2. Véase Alcảntara (1991 y 1992).

3. Véase O'Donnell (1994).

4. Sólo en Ecuador se pueden contabilizar en dos ocasiones consecutivas la sustitución del Presidente de la República por mecanismos no estrictamente constitucionales como aconteció en 1998 y en 2000 aunque la solución finalmente alcanzada tuviera un soporte constitucional al ser reemplazado el Presidente depuesto por el presidente del Congreso y por el Vicepresidente respectivamente.

5. Si bien en Panamá no se consiguió como consecuencia de la derrota del proyecto de reforma constitucional realizado bajo el gobierno de Pérez Balladares en 1999.

6. Véase Morgenstern y Nacif (2002).

7. Los procesos de destitución, mediante una forma u otra de juicio político, de los presidentes Fernando Collor de Mello en Brasil, Carlos Andrés Pérez en Venezuela, Raúl Cubas en Paraguay, Abdalá Bucaram de Ecuador, sin que por ello el sistema se quebrara, son una prueba del correcto funcionamiento de las instituciones. También lo fue la solución dada por el Congreso guatemalteco a la crisis abierta por el presidente Jorge Serrano. Véase Pérez Liñan (2000). Ernesto Samper fue igualmente sometido a juicio politico aunque quedó exonerado de la acusación vertida contra él de haber recibido fondos del narcotráfico para su campaña electoral presidencial. Pero asimismo las crisis politicas que afectaron a los Poderes Ejecutivos se resolvieron con una notable operatividad sin que se quebrara el orden constitucional como aconteció en los procesos de sustitución presidencial reguiar de Jamil Mahuad en Ecuador, Alberto Fujimori en Perú y Fernando de la Rúa en Argentina, acontecidos todos ellos durante 2001, el año de mayor inestabilidad en la región de los últimos tiempos, 0 en la superación de la crisis como ocurrió tras el fracaso de los golpes de Estado de Paraguay contra Juan Carlos Wasmosy en abril de 1996 y contra Hugo Chávez en Venezuela en abril de 2002.

8. A las que se podria añadir la propuesta por el presidente Uribe de Colombia.
9. El caso más evidente es el de México mediante la potenciación de la figura del Instituto Federal Electoral.

10. Véase Molina (2000).

11. Véase Alcántara (2001).

12. Uno de los casos de análisis más interesante es el mexicano puesto que la Constitución de 1917 suponía un modelo "duro" de economia fuertemente nacionalizada y estatizada que, no obstante, fue desmantelado en distintas etapas a lo largo de la década de 1990.

13. Véase Jordana (2001).

14. Las reformas tendentes a la primera elección popular de alcaldes en Colombia en 1989 fueron coetáneas de las que llevaron a la elección de gobernadores en Venezuela. Más adelante las reformas constitucionales de Colombia, Argentina y México propiciaron la elección de los gobernadores de los Departamentos en el primero, y de los alcaldes de la Capital Federal argentina y del regente del Distrito Federal en México.

15. Como es el caso de la Constitución ecuatoriana de 1998.

16. El caso más evidente es el de Colombia, donde el propio Estado ha concedido una "zona de despeje" a la guerrilla y donde diferentes actores no estatales Ilegan a controlar cerca del cuarenta por ciento del territorio nacional. Pero igualmente cabría referirse a Perú, Brasil y, en menor medida, México. En los dos primeros paises carteles de la droga y traficantes madereros imponen su ley ante la ausencia del Estado. En México, la guerrilla zapatista tiene un santuario en la Sierra Lacandona que inhibe la acción del Estado.

17. Véase Méndez et al. (1999).

18. Sobre el contenido del mismo puede verse Williamson (1994: 26-28)

19. Al iniciarse el siglo xxi América Latina contaba con líderes de la talla de Fernando H. Cardoso, Fidel Castro, Hugo Chávez y Vicente Fox cuyo grado de heterogeneidad y de disparidad en su talante, personalidad, ideología y vinculación partidista era evidente.

20. Con la Unión Europea, y a pesar del impulso que supuso en las relaciones entre ambas partes el ingreso de España y de Portugal en 1986, entre 1980 y 2000 la 
parte del comercio latinoamericano que representa la Unión Europea cayó del 20 al 15 por ciento y, lo que era más grave, en el año 2000 las importaciones europeas procedentes de América Latina apenas si representaban el 4,7 por ciento del total importado por la Unión Europea y las exportaciones a América Latina alcanzaban un exiguo 5,8 por ciento del total exportado, siendo cinco años antes estos porcentajes del 5,2 por ciento y del 5,7 por ciento respectivamente.

21. El Plan Colombia sería el ejemplo más evidente como "producto estrella" de la Administración norteamericana para con América Latina.

22. Véase O’Donnell (2002: 43).

23. La encuesta Fitzgibbon se lleva a cabo desde 1945. La metodología de la misma se basa en la clasificación de los paises latinoamericanos de acuerdo con quince criterios por parte de distinguidos académicos latinoamericanistas que son adecuados en niveles de medida ordinales y de intervalo. La encuesta de 2000 contó con la participación de 103 especialistas.

\section{Véase Easton (1975).}

25. Se trata de los resultados de una investigación llevada a cabo en el seno del Instituto Interuniversitario de Estudios de Iberoamérica y Portugal de la Universidad de Salamanca desde 1994 referida a las actitudes, valores y opiniones de los diputados latinoamericanos y que ha contado con el apoyo del Programa Nacional de Ciencia y Tecnología y otras entidades públicas y privadas.

26. Debe tenerse en cuenta a efectos metodológicos la circunstancia de coincidir dos ámbitos muestrales diferentes como son, por una parte, los ciudadanos y, por otra, los diputados.

27. Ver a este respecto el Indice de Confianza en el Gobierno (ICG) elaborado por la Universidad Torcuato Di Tella de Buenos Aires (htto://www.utdt.edu). El ICG argentino pasa, en una escala de 0 a 5 del 1,36 en enero de 2002 a 0,42 en agosto del mismo año.

28. El Grupo de Opinión Pública de la Universidad de Lima evaluaba en el 74,2 por ciento la desaprobación ciudadana a la gestión de Alejandro Toledo en el barómetro de agosto (http://www.ulima.edu.pe/).

29. De acuerdo con el lindice de Percepción de Corrupción (IPC) elaborado y publicado el 28.8.02 por Transparency International (http:/www.transparency.org) en una escala de diez puntos los paises latinoamericanos obtuvieron las siguientes puntuaciones: Chile: 7,5; Uruguay: 5, 1; Costa Rica: 4,5; Brasil: 4,0; Perú: 4,0; Colombia: 3,6; México: 3,6; R. Dominicana: 3.5; El Salvador: 3,4; Panamá: 3,0; Argentina: 2,8; Honduras: 2,7;

Guatemala: 2,5; Nicaragua: 2,5; Venezuela: 2,5; Bolivia: 2,2; Ecuador: 2,2 y Paraguay: 1,7 .

30. Expresión usada por Linz (1990: 29) para referirse a una concepción minimalista de la consolidación democrática.

31. Véase el desarrollo de estos puntos en Mainwaring (1999).

32. Se entiende por capital politico la presencia de confianza politica y en las instituciones, de disponibilidad cívica y compromiso político. Véase Mateos (2002: 153).

33. Véase Tanaka (1999: 16).

34. Véase Kenney (1997) y Mayorga (1995). 


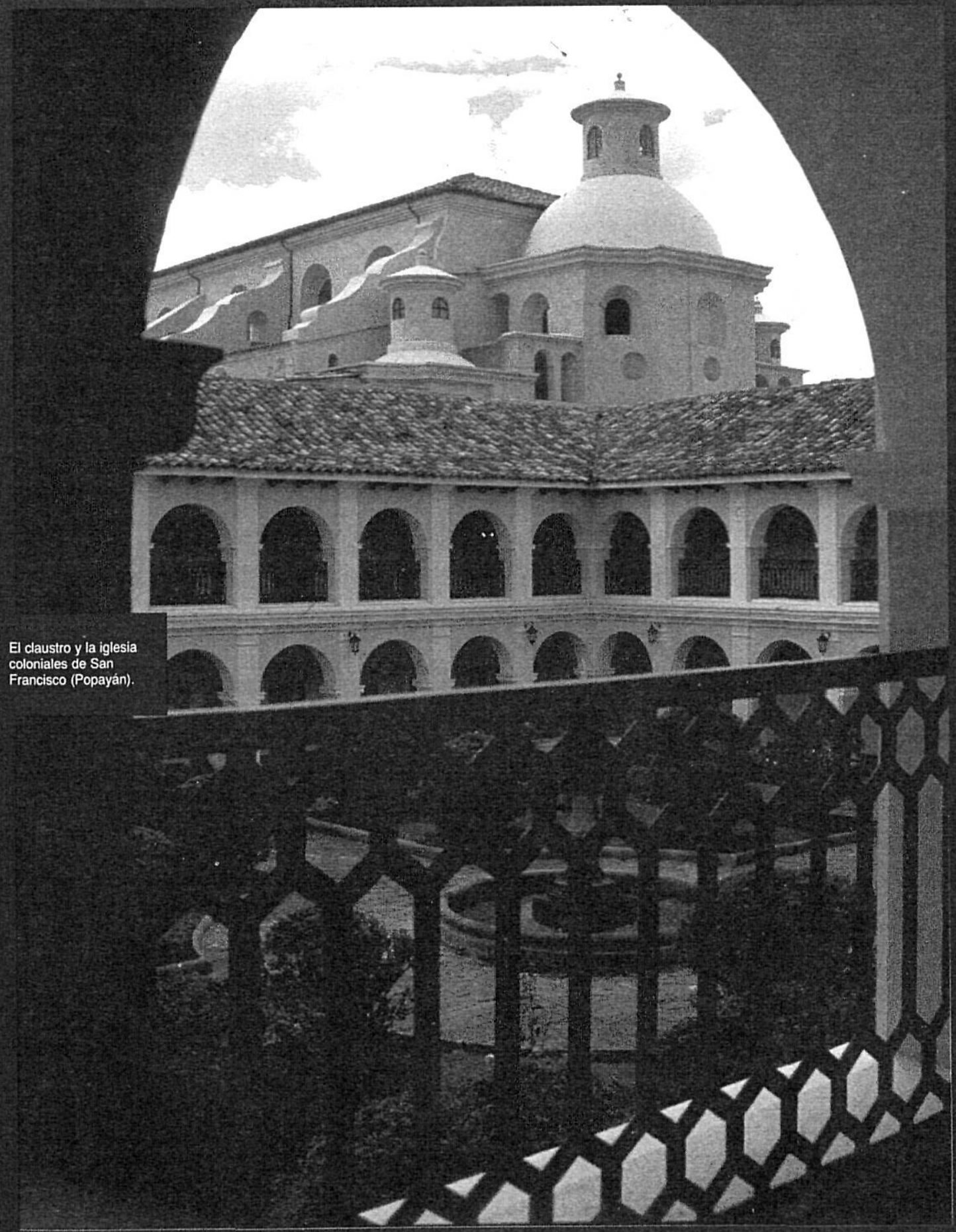

